\title{
Epitaxial self-alignment: A new route to hybrid active plasmonic nanostructures
}

\author{
Richard Nötzel , Adam Urbańczyk
}

\section{A R T I C L E I N F O}

Keywords:

III-V semiconductors

Quantum dot

Lateral ordering

Metal nanostructures

Plasmonics

Enhanced emission

Enhanced absorption

\begin{abstract}
A B S T R A C T
Concepts of lateral ordering of epitaxial semiconductor quantum dots (QDs) are for the first time transferred to hybrid nanostructures for active plasmonics. We review our recent research on the selfalignment of epitaxial nanocrystals of In and $\mathrm{Ag}$ on ordered one-dimensional $\operatorname{In}(\mathrm{Ga}) \mathrm{As}$ QD arrays and isolated QDs by molecular beam epitaxy. By changing the growth conditions the size and density of the metal nanocrystals are easily controlled and the surface plasmon resonance wavelength is tuned over a wide range in order to match the emission wavelength of the QDs. Photoluminescence measurements reveal large enhancement of the emitted light intensity due to plasmon enhanced emission and absorption down to the single $Q D$ level.
\end{abstract}

\section{Introduction}

Epitaxial semiconductor quantum dots (QDs) have enabled significant improvements of the performance of optoelectronic devices such as semiconductor lasers and optical amplifiers [1]. The QDs that are almost entirely employed are strain induced islands formed in the Stranski-Krastanov growth mode of lattice mismatched systems such as $\operatorname{InAs} / \mathrm{GaAs}$ and $\operatorname{InAs} / \mathrm{InP}$ and they are distributed randomly. For future quantum functional devices laterally ordered arrangements of QDs are required allowing operation down to the level of single electrons and photons with well controlled quantum mechanical and electromagnetic interactions [2]. There are numerous approaches to fabricate such ordered QD arrangements based on self assembly and growth on patterned substrates and a rather complete collection can be found in [3]. Over the years we have contributed to this field with various novel approaches and concepts, including periodic self-faceting on highindex surfaces [4], self-organization of strained quantum disks [5], natural self-faceting on patterned substrates [6], self-organized anisotropic strain engineering [7], guided and directed selforganization on patterned substrates [8], and shape controlled selective area growth [9].

Now we made a significant step forward to transfer concepts of self-organized ordering to hybrid nanostructures, namely the selfalignment of epitaxial metal nanocrystals to ordered semiconductor QD arrays and single QDs to add plasmonic functionalities. In the following we discuss our three main achievements, critically discuss our observations and interpretations, bring our work in relation to the state-of-the-art, and conclude.

\section{Research highlights}

First, we have shown that In nanocrystals can be grown epitaxially on GaAs [10], in this case by molecular beam epitaxy (MBE). They exhibit clear faceting and a well defined epitaxial relationship with the substrate as determined from atomic force microscopy (AFM) and reflection high-energy electron diffraction (RHEED) studies, see Fig. 1a-d. Formation of epitaxial metal nanocrystals on semiconductor surfaces is actually not uncommon and has been reported long ago in the $\mathrm{Ag} / \mathrm{Si}$ materials system [11]. We found, however, that due to the epitaxial growth their size and density are easily controlled by the growth conditions, i.e., growth temperature, and growth rate and amount. Higher growth temperature results in larger size and lower density, higher growth rate results in smaller size and higher density, and larger In amount results in larger size and higher density, see Fig. 1a-c, just the same way as known for InAs QDs. Accordingly, the surface plasmon resonance (SPR) of the In nanocrystals, determined from differential reflectivity (DR) measurements at room temperature, can be tuned over a wide wavelength range, shown in Fig. 1e, covering the emission wavelengths of epitaxial semiconductor QDs.

Second, we have shown that the In nanocrystals self-align to InAs/GaAs QDs in a very similar manner as stacked QDs align in growth direction due to vertical strain correlation [12,13], but this type of alignment has never before been applied to hybrid systems. The self-alignment has been clearly demonstrated by the ordered growth of In nanocrystals on ordered one-dimensional InGaAs/ 

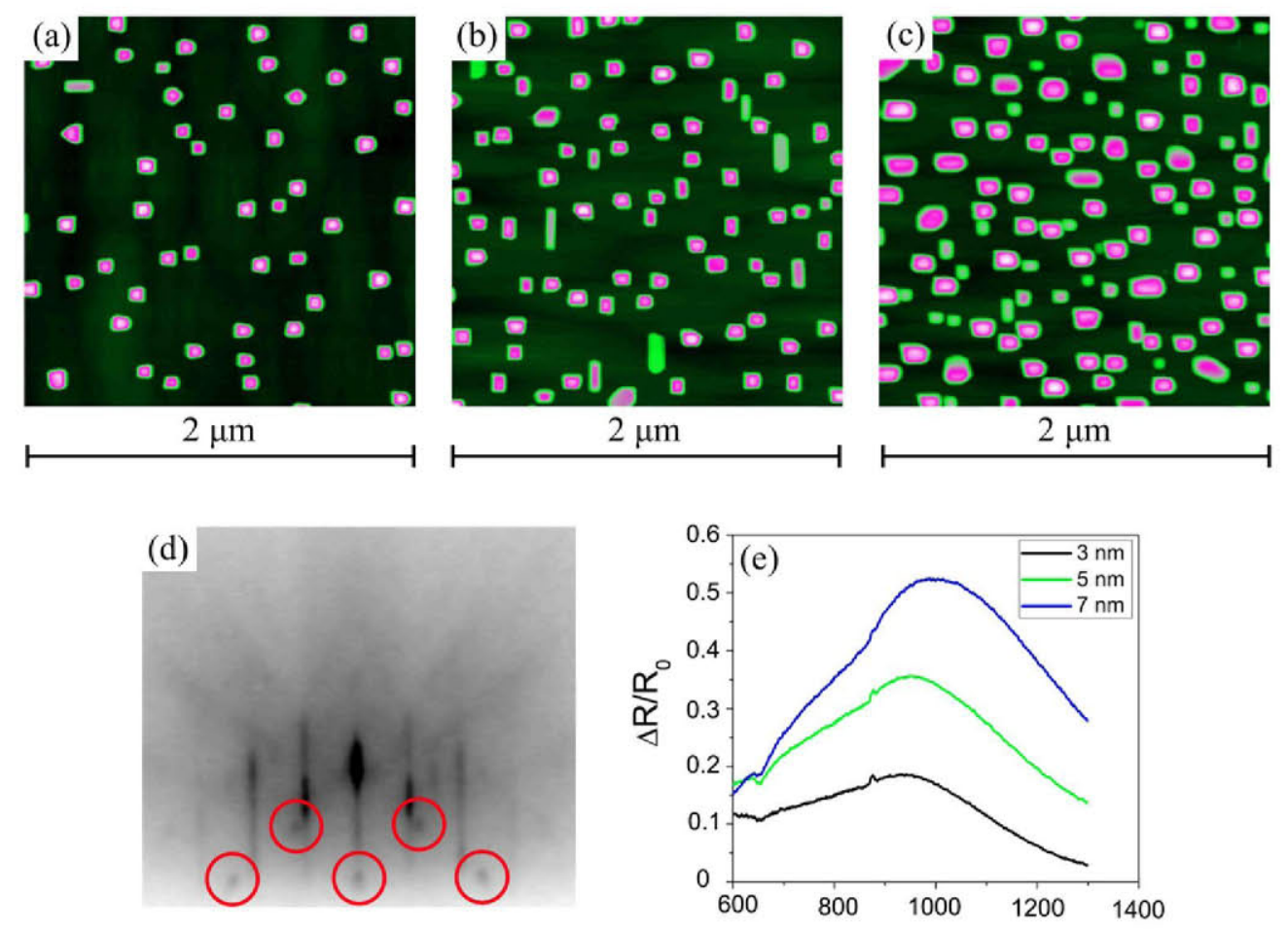

Fig. 1. AFM images of In nanocrystals grown by MBE on GaAs (100) with coverage of (a) 3 , (b) 5 , and (c) 7 nm. The growth temperature is $100{ }^{\circ} \mathrm{C}$ and the growth rate is $0.008 \mathrm{~nm} / \mathrm{s}$. Some nanocrystals are elongated in the [011] direction. All scan fields are $2 \times 2 \mu \mathrm{m}^{2}$. (d) Typical RHEED pattern after In deposition in the [011] GaAs direction. The red circles indicate the In transmission diffraction spots. (e) Differential reflectivity spectra taken at room temperature of the In nanocrystals with 3,5 , and $7 \mathrm{~nm}$ coverage.

GaAs QD arrays, shown in Fig. 2a [14]. These one-dimensional QD arrays are formed by self-organized anisotropic strain engineering of an InGaAs/GaAs superlattice template, a concept for QD ordering which we have developed earlier [7]. Importantly, the self-alignment of the In nanocrystals is sustained in the presence of a thin GaAs cap layer on the QDs. Here, the thickness is $3 \mathrm{~nm}$. In this way the lateral and vertical positioning of the In nanocrystals with respect to the QDs is controlled with nanometer-scale precision. Laterally due to the self-alignment and vertically by the thin GaAs cap layer on the QDs.

This unprecedented position control has allowed the observation of plasmon enhanced emission down to the single QD level $[14,15]$. Plasmon enhanced emission relies on the confinement of light at deep sub wavelength length scales and the corresponding huge local optical near-field enhancements due to the excitation of localized surface plasmons in the metal nanocrystals. Therefore, the metal nanocrystal - QD separation is most crucial as for too small separation the $\mathrm{QD}$ emission is quenched due to nonradiative energy transfer while for too large separation the plasmonic effects will be lost, especially in the high-refractive index semiconductors where the optical near-field decays already within a few nanometers. Fig. 2b shows the photoluminescence (PL) spectra taken at $140 \mathrm{~K}$ and room temperature of the $\mathrm{QD}$ arrays with In nanocrystals and the PL spectrum of a reference sample without In nanocrystals taken at room temperature. Precisely spectrally overlapping with the SPR the QD PL is enhanced in the presence of the In nanocrystals while no such enhancement is observed without In nanocrystals or out of resonance. This characteristic change in the spectral shape of the PL clearly indicates enhanced QD emission in contrast to enhanced absorption which would affect all QDs close to the In nanocrystals equally, enhancing the overall emission. The PL enhancement is observed up to room temperature, though more pronounced at lower temperature due to stronger SPRs for lower resistive losses. In micro-PL, shown in Fig. 2c, the enhanced PL splits into distinct sharp peaks due to emission from single QDs. This can be resolved due to the intentionally low density of the In nanocrystals resulting in plasmon enhanced emission from only a few QDs, thus opening the door to quantum optical studies and applications at nanoscale dimensions.

Third, we have shown that also Ag nanocrystals can be grown epitaxially on GaAs with excellent tunability of the structural properties and plasmon resonances and they self-align to InAs/ GaAs QDs [16]. This is important as Ag is the material of choice for plasmonics, exhibiting the lowest resistivity of all metals in the wavelength range of interest and, moreover, it shows the generality of our approach of epitaxial self-alignment. The $\mathrm{Ag}$ nanocrystals self-align right on top of the InAs/GaAs QDs, in this case demonstrated for dilute near surface QDs capped with $3 \mathrm{~nm}$ GaAs to control the vertical separation, shown in the AFM image in Fig. 3a. The red rectangles are the $\mathrm{Ag}$ nanocrystals on the capped InAs/GaAs QDs protruding underneath. The inset shows a reference capped single $\mathrm{QD}$ without Ag nanocrystal for comparison. As seen, the probability of alignment is $100 \%$ as the density of the $\mathrm{Ag}$ nanocrystals is perfectly matched to that of the QDs. The Ag nanocrystals are often elongated. Interestingly, the direction of elongation is rotated on the QDs compared to that when the Ag nanocrystals are grown on bare GaAs. This provides a first hint to template based shape control of noble-metal nanocrystals in addition to the control of size and density through other growth parameters like growth temperature, growth rate, and deposition amount.

Due to the elongation of the Ag nanocrystals the SPR response exhibits a strong linear polarization dependence with large peak separations for light polarized parallel and perpendicular to the long axis, shown in Fig. 3b. Most important, the presence of the Ag nanocrystals results in a large intensity enhancement of the QD PL. In micro-PL, particular lines from individual QDs are much stronger which is never observed without $\mathrm{Ag}$ 

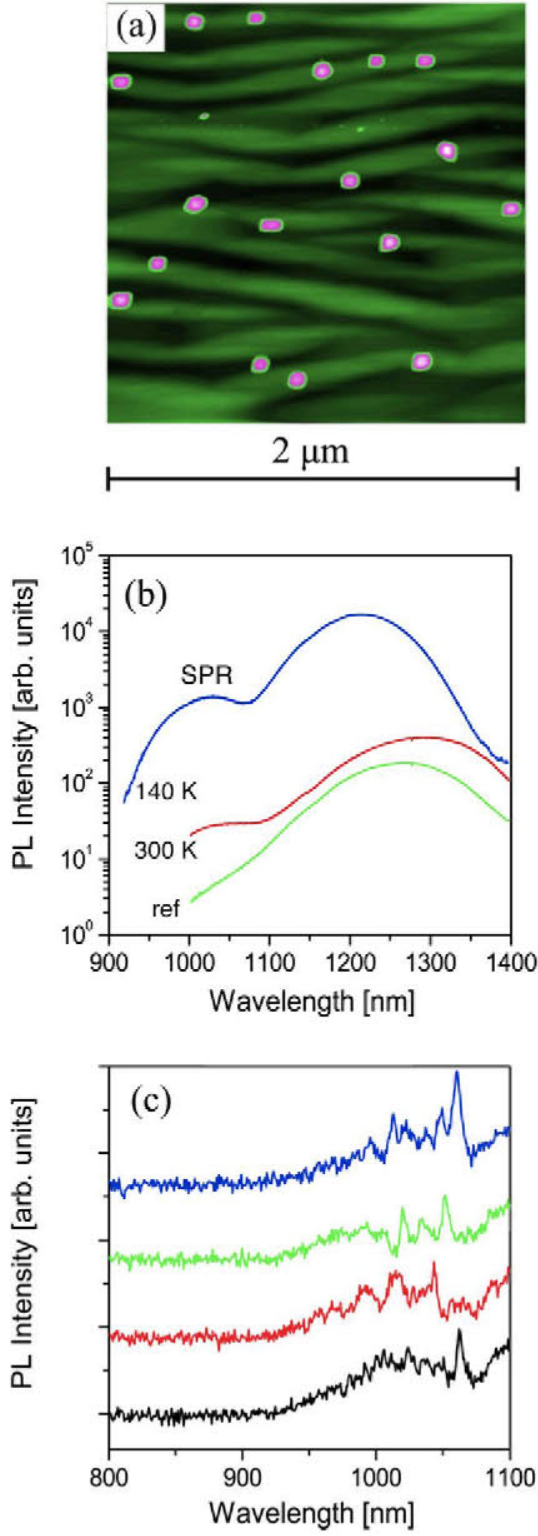

Fig. 2. (a) AFM image of In nanocrystals (purple rectangles) self-aligned on onedimensional InGaAs/GaAs QD arrays (light green stripes) formed by self-organized anisotropic strain engineering. Growth is by MBE. The In amount is 4 monolayers and the growth temperature is $120^{\circ} \mathrm{C}$. The $\mathrm{OD}$ arrays run along [0-11] and are capped by $3 \mathrm{~nm}$ GaAs. The scan field is $2 \times 2 \mu \mathrm{m}^{2}$. (b) PL spectra taken at $140 \mathrm{~K}$ and room temperature of the QD arrays with In nanocrystals and PL spectrum of a reference sample without In nanocrystals taken at room temperature. SPR indicates the wavelength range of the surface plasmon resonance of the In nanocrystals determined by differential reflectivity measurements. (c) Micro-PL spectra of the QD arrays with In nanocrystals taken at $140 \mathrm{~K}$ at four different positions in the spectral range of the SPR.

nanocrystals, shown in Fig. 3c. Here we attribute the majority of the PL intensity enhancement to near-field enhanced absorption of the exciting laser light, also in resonance with the SPR, and thus greater carrier injection into $\mathrm{GaAs}$ and the QDs where the alignment is essential. This is because there is no clear spectral correspondence of the enhanced PL intensity with the SPR and, moreover, a similar intensity enhancement is observed also for the PL of bulk GaAs where the excited carriers are delocalized. Importantly, in view of applications, this reveals the feasibility of plasmon enhanced ultra-thin film and nanostructure solar cells.
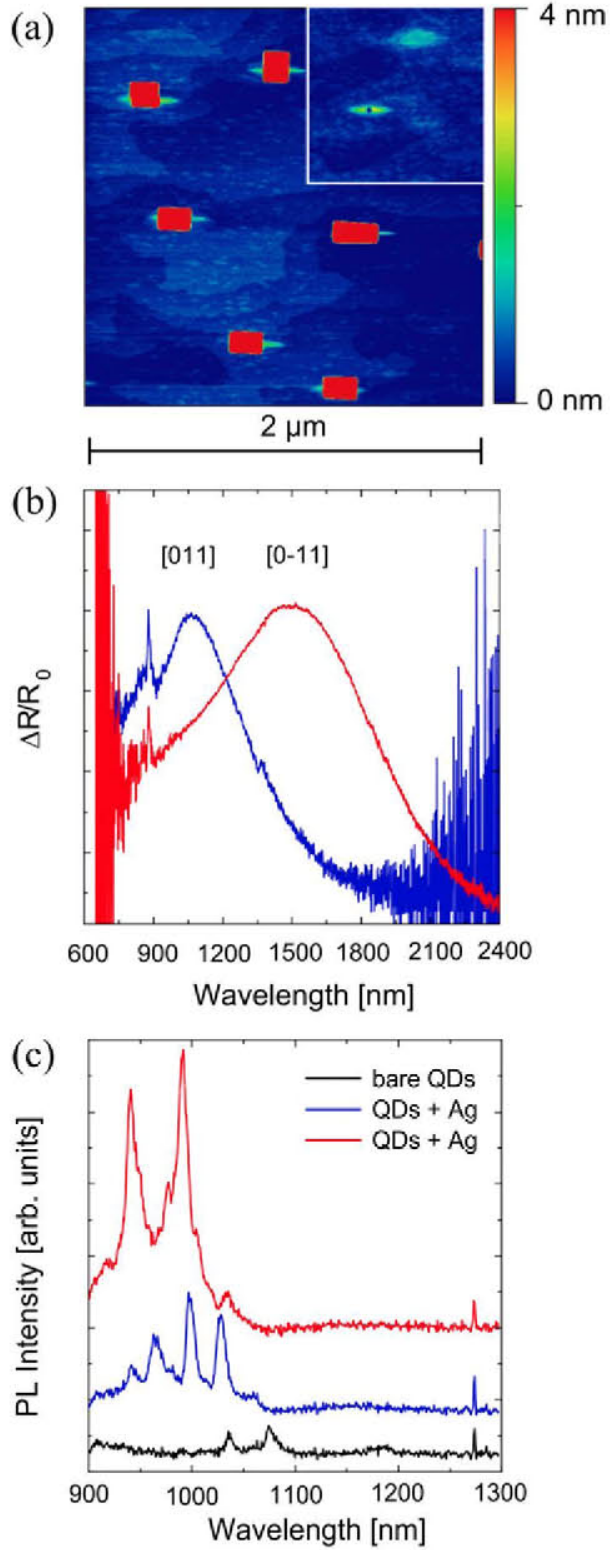

Fig. 3. (a) AFM image of Ag nanocrystals (red rectangles) self-aligned on top of InAs/GaAs QDs capped with $3 \mathrm{~nm}$ GaAs protruding underneath. Growth is by MBE. The $\mathrm{Ag}$ amount is $0.5 \mathrm{~nm}$ and the growth temperature is $400^{\circ} \mathrm{C}$. The nanocrystal elongation is along [0-11]. The scan field is $2 \times 2 \mu \mathrm{m}^{2}$. Inset: capped single InAs/ GaAs OD without Ag nanocrystal for comparison. The scan field is $780 \times 780 \mathrm{~nm}^{2}$. (b) Differential reflectivity spectra for light polarized along [011] and [0-11] of the sample with Ag nanocrystal-QD hybrids. (c) Low-temperature micro-PL spectra of the QDs of the metal-QD hybrid structures taken at two different positions and of a reference sample with only near-surface QDs.

\section{Critical discussion of our observations of the optical properties and interpretation}

As the field of active nanoplasmonics, the way we approach it here, is at a quite early stage, it bears many risks of misconceptions and misinterpretations. Therefore, we give here a critical discussion of our spectroscopic results and their interpretation.

In and $\mathrm{Ag}$ are from a spectroscopic point of view very different metals. The imaginary part of the dielectric function of In is significantly larger than that of $\mathrm{Ag}$ resulting in much stronger damping effects. This translates into a SPR which should be broader by around one order of magnitude [17]. This is true 
when considering the intrinsic properties of In and Ag nanoparticles. However, in our experiments, we study the optical response of In and Ag nanocrystal ensembles with considerable size distribution. Hence, the widths of the SPRs are determined by inhomogeneous broadening which likely makes them comparable. Additionally one needs to take into account that the contribution due to resistive losses to the intrinsic SPR width is not given by the imaginary part of the dielectric function alone and that radiation damping becomes significant for the particle sizes under consideration [18]. It is well known that not only Ag but also In nanoparticle ensembles support well defined SPRs of comparable widths [19-21].

Plasmon resonances of metals are commonly associated to be in the visible spectral range in contrast to our observations where they appear in the near-infrared. However, considering the fact that the In and $\mathrm{Ag}$ nanocrystals are positioned on high-refractive index GaAs and additionally often exhibit a strong elongation, especially the Ag nanocrystals, such long wavelength SPRs seem realistic and necessary to spectrally match them with the QD emission upon independent adjustment of the size of the In and $\mathrm{Ag}$ nanocrystals. Very simple estimates based on effective refractive index considerations support that, but unfortunately this is not straight-forward to calculate accurately. Here, it is worth mentioning that many groups have reported a wide wavelength tunability of the SPRs of Ag nanoparticles by engineering the size, shape $[22,23]$, and dielectric environment [24], extending even into the mid-infrared spectral region.

Technically it is not easy to measure DR spectra in the presence of high-refractive index substrates. There may occur many experimental artifacts, such as due to strong back reflection of the substrate which could result in peaks when the reference is slightly misaligned. However, we observe only a slowly varying background when the reference is intentionally misaligned. Moreover, differential transmittance measurements, which have also been performed, reveal the same resonance positions as the DR spectra. Most important, our measurements are consistent in two crucial points: First, the shift to longer wavelengths of the resonances with increasing In and $\mathrm{Ag}$ nanocrystal size and second the linear polarization behavior upon nanocrystal elongation.

A further issue is the need for near-surface QDs due to the rapid decay of the optical near field. This leads to a strong interaction of the QDs with surface defects which manifests itself in the rather broad single $\mathrm{QD}$ emission of a few meV. Moreover, this interaction leads to a significant quenching of the $\mathrm{QD}$ emission. The presence of a metal particle close by may simply change the state of the defects, change the band structure, and lead to a reduced quenching, thereby enhancing the QD emission. But this would, as enhanced absorption, affect all QDs similarly and not enhance the emission of only those QDs which are in resonance with the SPR, as we observe for the case of the In nanocrystals. In all, it is evident, that substantial support from theory is needed for the interpretation of spectroscopic results as presented here for hybrid semiconductor QD - metal nanostructures, which we seek to come.

\section{State-of-the-art}

Now we relate our experimental work to the state-of-the-art of the field. Position control of active plasmonic nanostructures has been demonstrated by applying conventional approaches like the scanning of a metallic tip over a QD or the manipulation of an emitter or metal particle by a scanning probe tip $[25,26]$. Approaches based on self-assembly exclusively employed colloidal QDs and metal nanoparticles or structured metal films [27-31]. The QDs and metal nanoparticles or films were separated either by dielectric spacer layers or organic molecules, most prominent DNA molecules. Enhanced emission and enhanced absorption have been observed in such structures, also down to the single QD level. However, these structures are not easy to integrate in wafer based scalable devices and therefore impractical for most applications. Epitaxial structures naturally overcome this drawback.

\section{Conclusions}

In conclusion, In and Ag nanocrystals were grown epitaxially on GaAs by MBE. Their size and density were tuned by varying the substrate temperature, coverage, and growth rate resulting in strong shifts of the SPRs. When deposited on $\operatorname{In}(\mathrm{Ga})$ As $\mathrm{QD}$ arrays or isolated QDs the In and $\mathrm{Ag}$ nanocrystals self-aligned on top of the QDs. PL measurements revealed clear intensity enhancements which were attributed to SPR enhanced emission and absorption. The superior control of size, density, and position demonstrated here for epitaxial metal nanocrystals is highly relevant to exploit plasmonic effects for a much wider range of applications than discussed so far, including sensing [32,33], spectroscopy [34], imaging [35], solar energy conversion [36,37], nanophotonic circuits [38,39], and many others. It should be emphasized here that metal nanostructures when they are single-crystalline exhibit the best plasmonic properties [40].

An outstanding challenge that must be addressed is shape control of the metal nanocrystals to fully exploit plasmonic functionality. In principle, conventional direct patterning by, e.g., electron beam lithography or focused ion beam milling seems more flexible in this respect. We anticipate however, that methods based on direct growth, most probably in combination with lithographic patterning will be more feasible, in particular when structural perfection and alignment are concerned. Then more optical properties of QDs can be controlled such as polarization $[41,42]$ and directionality $[43,44]$ of the emitted light. This will lead to novel truly nanophotonic applications with electronic and optical fields fully controlled and confined to the nanoscale, including efficient single photon sources, optical transistors [45], nanolasers [46,47] and even provide the basis for quantum information processing when the strong coupling regime can be reached $[48,49]$. We believe that this is at realistic reach. The epitaxial growth of metal nanocrystals follows astonishingly close the well known mechanisms governing the growth of semiconductor nanostructures which have been studied over many years to have better and better control over the structural and optical properties, and we have only just started with metals.

\section{References}

[1] Coleman JJ, Young JD, Garg A. Semiconductor quantum dot lasers: a tutorial. J Lightwave Technol 2011;29:499-510.

[2] Nötzel R. Quantum dots for future nanophotonic devices: lateral ordering, position, and number control. IEEE Phot J 2010;2:67-80.

[3] Lateral alignment of epitaxial quantum dots. 2007 Schmidt OG Ed. Springer Series on Nanoscience and Technology ISBN: 978-3-540-46935-3.

[4] Nötzel R, Ledentsov NN, Däweritz L, Hohenstein M, Ploog K. Direct synthesis of corrugated superlattices on non-(100)-oriented surfaces. Phys Rev Lett 1991;67:3812-5.

[5] Nötzel R, Temmyo J, Tamamura T. Self-organized growth of strained InGaAs quantum disks. Nature 1994;369:131-3.

[6] Nötzel R, Niu ZC, Ramsteiner M, Schönherr HP, Trampert A, Däweritz L, et al. Uniform quantum-dot arrays formed by natural self-faceting on patterned substrates. Nature 1998;392:56-9.

[7] Mano T, Nötzel T, Hamhuis GJ, Eijkemans TJ, Wolter JH. Formation of InAs quantum dot arrays on GaAs (100) by self-organized anisotropic strain engineering of a (In,Ga)As superlattice template. Appl Phys Lett 2002;81:1705-7

[8] Selcuk E, Silov AY, Nötzel R. Single InAs quantum dot arrays and directed selforganization on patterned GaAs (311)B substrates. Appl Phys Lett 2009;94:263108

[9] Wang H, Yuan J, Rieger T, van Veldhoven PJ, Nouwens P, Eijkemans TJ, et al. Distribution control of $1.55 \mu \mathrm{m}$ InAs quantum dots down to small numbers on 
truncated InP pyramids grown by selective area metal organic vapor phase epitaxy. Appl Phys Lett 2009;94:143103.

[10] Urbañczyk A, Hamhuis GJ, Nötzel R. In islands and their conversion to InAs quantum dots on GaAs (100): structural and optical properties. J Appl Phys 2010;107:014312.

[11] Hanbücken $M$, Futamoto $M$, Venables JA. Nucleation, growth and the intermediate layer in $\mathrm{Ag} / \mathrm{Si}(100)$ and $\mathrm{Ag} / \mathrm{Si}$ (111). Surf Sci 1984:147:433-50.

[12] Xie QH, Madhukar A, Chen P, Kobayashi NP. Vertically self-organized InAs quantum box islands on GaAs (100). Phys Rev Lett 1995;75:2542-5.

[13] Tersoff J, Teichert C, Lagally MG. Self-organization in growth of quantum dot superlattices. Phys Rev Lett 1996;76:1675-8.

[14] Urbańczyk A, Hamhuis GJ, Notzel R. Strain-driven alignment of In nanocrystals on InGaAs quantum dot arrays and coupled plasmon-quantum dot emission. Appl Phys Lett 2010;96:113101.

[15] Urbańczyk A, Hamhuis GJ, Nötzel R. Coupling of single InGaAs quantum dots to the plasmon resonance of a metal nanocrystal. Appl Phys Lett 2010;97:043105.

[16] Urbańczyk A, van Otten FWM, Nötzel R. Self-aligned epitaxial metalsemiconductor hybrid nanostructures for plasmonics. Appl Phys Lett 2011;98:243110.

[17] Zeman EJ, Schatz GC. An accurate electromagnetic theory study of surface enhancement factors for silver, gold, copper, lithium, sodium, aluminum, gallium, indium, zinc, and cadmium. J Phys Chem 1987;91:634-43.

[18] Hu M, Novo C, Funston A, Wang H, Staleva H, Zou S, et al. Dark-field microscopy studies of single metal nanoparticles: understanding the factors that influence the linewidth of the localized surface plasmon resonance. J Mater Chem 2008;18:1949-60.

[19] Dragan Al, Geddes CD. Indium nanodeposits: a substrate for metal-enhanced fluorescence in the ultraviolet spectral region. J Appl Phys 2010;108:094701.

[20] Li H, Liang C, Liu M, Zhong K, Tong Y, Liu P, et al. Synthesis of indium nanowires by galvanic displacement and their optical properties. Nanoscale Res Lett $2009 ; 4: 47-53$.

[21] Cingarapu S, Yang Z, Sorensen CM, Klabunde KJ. Synthesis of indium nanoparticles: digestive ripening under mild conditions. Inorg Chem 2011;50:5000-5.

[22] Mock JJ, Barbic M, Smith DR, Schultz DA, Schultz S. Shape effects in plasmon resonance of individual colloidal silver nanoparticles. J Chem Phys $2002 ; 116: 6755-9$.

[23] Wiley B], Chen Y, McLellan JM, Xiong Y, Li ZY, Ginger D, et al. Synthesis and optical properties of silver nanobars and nanorice. Nano lett 2007;7:1032-6.

[24] Jensen TR, Duval Malinsky $M$, Haynes $C L$, Van Duyne RP. Nanosphere lithography: tunable localized surface plasmon resonance spectra of silver nanoparticles. J Phys Chem B 2000;104:10549-56.

[25] Farahani JN, Pohl DW, Eisler HJ, Hecht B. Single quantum dot coupled to a scanning optical antenna: a tunable superemitter. Phys Rev lett 2005;95:017402.

[26] Pfeiffer M, Lindfors K, Wolpert C, Atkinson P, Benyoucef M, Rastelli A, et al. Enhancing the optical excitation efficiency of a single self-assembled quantum dot with a plasmonic nanoantenna. Nano Lett 2010:10:4555-8.

[27] Kulakovich O, Strekal N, Yaroshevich A, Maskevich S, Gaponenko S, Nabiev I, et al. Enhanced luminescence of CdSe quantum dots on gold colloids. Nano Lett 2002;2:1449-52.

[28] Pons T, Medintz IL, Sapsford KE, Higashiya S, Grimes AF, English DS, et al. On the quenching of semiconductor quantum dot photoluminescence by proximal gold nanoparticles. Nano Lett 2007;7:3157-64.
[29] Kramer RK, Pholchai N, Sorger VJ, Yim TJ, Oulton R, Zhang X. Positioning of quantum dots on metallic nanostructures. Nanotechnology 2010;21: 145307.

[30] Maye MM, Gang O, Cotlet M. Photoluminescence enhancement in CdSe/ZnSDNA linked-Au nanoparticle heterodimers probed by single molecule spectroscopy. Chem Commun 2010;46:6111-3.

[31] Vion C, Spinicelli P, Coolen L, Schwob C, Frigerio JM, Hermier JP, et al. Controlled modification of single colloidal CdSe/ZnS nanocrystal fluorescence through interactions with a gold surface. Opt Express 2010;18:7440-55.

[32] Homola J. Surface plasmon resonance sensors for detection of chemical and biological species. Chem Rev 2008;108:462-93.

[33] Anker JN, Hall WP, Lyandres O, Shah NC, Zhao J, van Duyne RP. Biosensing with plasmonic nanosensors. Nature Mater 2008;7:442-53.

[34] Biswas A, Wang T, Biris AS. Single metal nanoparticle spectroscopy: optical characterization of individual nanosystems for biomedical applications. Nanoscale 2010;2:1560-72.

[35] Linman M], Abbas A, Cheng QA. Interface design and multiplexed analysis with surface plasmon resonance (SPR) spectroscopy and SPR imaging. Analyst 2010;135:2759-67.

[36] Catchpole KR, Polman A. Plasmonic solar cells. Opt Express 2008:16:21793-800.

[37] Ferry VE, Munday JN, Atwater HA. Design considerations for plasmonic photovoltaics. Adv Mater 2010;22:4794-808.

[38] Maier SA, Atwater HA. Plasmonics: localization and guiding of electromagnetic energy in metal/dielectric structures. J Appl Phys 2005;98:011101.

[39] Ebbesen TW, Genet C, Bozhevolnyi Sl. Surface-plasmon circuitry. Phys Today 2008;61:44-50

[40] Huang JS, Callegari V, Geisler P, Brüning C, Kern J, Prangsma JC, et al. Atomically flat single-crystalline gold nanostructures for plasmonic nanocircuitry. Nat Commun 2010;1:150.

[41] Mertens H, Biteen JS, Atwater HA, Polman A. Polarization-selective plasmonenhanced silicon quantum-dot luminescence. Nano lett 2006;6:2622-5.

[42] Cheng MT, Liu SD, Wang QQ. Modulating emission polarization of semiconductor quantum dots through surface plasmon of metal nanorod. Appl Phys Lett 2008;92:162107.

[43] Curto AG, Volpe G, Taminiau TH, Kreuzer MP, Quidant R, van Hulst NF. Unidirectional emission of a quantum dot coupled to a nanoantenna. Science 2010;329:930-3.

[44] Kosako T, Kadoya Y, Hofmann HF. Directional control of light by a nano-optical Yagi-Uda antenna. Nat Phot 2010;4:312-5.

[45] Chang DE, Sorensen AS, Demler EA, Lukin MD. A single-photon transistor using nanoscale surface plasmons. Nat Phys 2007;3:807-12.

[46] Noginov MA, Zhu G, Belgrave AM, Bakker R, Shalaev VM, Narimanov EE, et al. Demonstration of a spaser-based nanolaser. Nature 2009;460:1110-U68.

[47] Bergman DJ, Stockman MI. Surface plasmon amplification by stimulated emission of radiation: quantum generation of coherent surface plasmons in nanosystems. Phys Rev Lett 2003;90:027402.

[48] Artuso RD, Bryantt GW. Optical response of strongly coupled quantum dot metal nanoparticle systems: double peaked fano structure and bistability. Nano Lett 2008;8:2106-11.

[49] Gomez DE, Vernon KC, Mulvaney P, Davis T]. Surface plasmon mediated strong exciton-photon coupling in semiconductor nanocrystals. Nano Lett $2010 ; 10: 274-8$ 